\title{
Can lymphangiosarcoma be resurrected? A clinicopathological and immunohistochemical study of lymphatic differentiation in 49 angiosarcomas
}

\author{
Cohra C Mankey, Jonathan B McHugh, Dafydd G Thomas \& David R Lucas \\ Department of Pathology, University of Michigan, Ann Arbor, MI, USA
}

Date of submission 18 November 2008

Accepted for publication 12 June 2009

Mankey C C, McHugh J B, Thomas D G \& Lucas D R

(2010) Histopathology 56, 364-371

\section{Can lymphangiosarcoma be resurrected? A clinicopathological and immunohistochemical study of lymphatic differentiation in 49 angiosarcomas}

Aims: The term lymphangiosarcoma has largely been abandoned in the current classification of endothelial neoplasms. Recently, a number of lymphatic-associated antibodies have been developed for immunohistochemistry, which frequently stain angiosarcomas, implying lymphatic or mixed lymphatic and blood vascular differentiation is common. The aim was to investigate further lymphatic antigen expression, and to explore the relation of immunohistochemistry to morphological and clinical findings.

Methods and results: Forty-nine angiosarcomas in tissue microarrays were analysed with D2-40 and antibodies to Prox-1 and vascular endothelial growth factor receptor (VEGFR)-3. D2-40 was positive in 53\%, Prox-1 in $76 \%$, and VEGFR-3 in 57\%. Tumours with features attributable to lymphatic differentiation such as hobnail and kaposiform morphologies were more often positive with these markers, including a statistical association between D2-40 and hobnailing. Ten tumours had features suggestive of lymphatic differentiation, namely well-differentiated histology, interanastomosing channels devoid of red cells, prominent hobnailing, lymphoid aggregates, and multi-antigen expression of D2-40 (100\%), Prox-1 (100\%) and VEGFR-3 (60\%), which might be deserving of the appellation lymphangiosarcoma. Nine were cutaneous scalp/facial tumours in elderly patients and one arose within chronic lymphoedema.

Conclusions: Lymphatic differentiation is common in angiosarcoma, certain subsets show greater lymphatic differentiation than others, and lymphangiosarcoma may be defined pathologically, rather than clinically.

Keywords: angiosarcoma, D2-40, immunohistochemistry, lymphangiosarcoma, podoplanin, Prox-1, VEGFR-3

Abbreviations: LYVE-1, lymphatic vessel hyaluronan receptor-1; TMA, tissue microarray; VEGFR, vascular endothelial growth factor receptor

\section{Introduction}

The concept of lymphangiosarcoma originated in 1948 when Stewart and Treves described six cases of a malignant vascular neoplasm that arose in the upper extremity many years after radical mastectomy. ${ }^{1}$ Because the neoplasm arose in the setting of chronic lymphoedema, it was presumed to have arisen from

Address for correspondence: Dr David R. Lucas, Department of Pathology, University of Michigan, 2G332 UH, Ann Arbor,

MI 48109-0054, USA. e-mail: drlucas@umich.edu lymphatic vessels, thus having lymphatic endothelial differentiation. Today pathologists rarely make this diagnosis, and distinguishing between lymphangiosarcoma and haemangiosarcoma has essentially become an obsolete practice. This is summarized in Enzinger and Weiss's Soft tissue tumors textbook: 'Since it is usually impossible to determine which tumors display lymphatic versus vascular differentiation, all are referred to as angiosarcoma, even those that arise in the setting of lymphedema'. ${ }^{2}$

Recently, a number of antigens preferentially expressed by lymphatic endothelial cells have been discovered. Examples include podoplanin (recognized 
by the monoclonal antibody D2-40), Prox-1, vascular endothelial growth factor receptor (VEGFR)-3, and lymphatic vessel hyaluronan receptor-1 (LYVE-1), all of which have been used to study vascular tumours by immunohistochemistry. These antigens are expressed in nearly all lymphangiomas, ${ }^{3-9}$ but in only a minority of haemangiomas. ${ }^{4,5,7-12}$ They are also expressed in nearly all Kaposi sarcomas, ${ }^{3-5,7,8,11,13}$ a tumour believed to have at least partial lymphatic differentiation. Data about expression of lymphatic antigens in angiosarcoma, however, are limited. ${ }^{3-5,8,11,14}$ For example, only one group has studied a large number of cases, ${ }^{4}$ only single antigens have been studied in all but one series, ${ }^{15}$ and Prox-1 has not been studied at all. Some studies suggest that most angiosarcomas show some degree of lymphatic differentiation, , ${ }^{3,4,14}$ whereas others suggest that certain subsets show lymphatic differentiation and suggest a link between expression of lymphatic antigens and morphological features such as hobnail cytology, ${ }^{5,11}$ non-epithelioid cytology, ${ }^{4,15}$ kaposiform architecture, ${ }^{11}$ and lymphoid infiltrates or aggregates. ${ }^{5,11}$ The purpose of our study was to analyse a large number of angiosarcomas by immunohistochemistry with D2-40, and antibodies to Prox-1 and VEGFR-3 to determine the extent of lymphatic antigen expression. In addition, we wanted to see if we could identify a subgroup of angiosarcomas that might be considered lymphangiosarcomas based upon morphological, immunohistochemical and clinical features.

\section{Materials and methods}

This study was conducted under approval of the Institutional Review Board of the University of Michigan. The pathology database was searched for all angiosarcomas diagnosed between 1986 and 2006. Forty-nine cases had sufficient tissue in paraffin blocks for immunohistochemical studies. Each case was classified as sporadic cutaneous, sporadic visceral/ deep-seated, lymphoedema-associated or radiationassociated based upon information gleaned from the medical records. Haematoxylin and eosin-stained sections were re-examined to confirm the diagnosis.
The following features were evaluated: differentiation (well, moderate or poor, based upon criteria of the French Federation of Cancer Centre Systems), architecture (vasoformative, fascicular/kaposiform or solid), cytology (hobnail, epithelioid, spindled or pleomorphic) and presence or absence of lymphoid aggregates within the tumour. Cuboidal cells with scant cytoplasm and apical hyperchromatic nuclei that protruded into the vascular lumen were considered hobnail cells, whereas cells with abundant eosinophilic cytoplasm and vesicular nuclei with prominent nucleoli were considered epithelioid cells. The series did not include any lowgrade malignant vascular tumour such as retiform haemangioendothelioma or Dabska tumour, tumours with frequent hobnailing.

Tissue microarrays (TMAs) with three 1.0-mm punches of each tumour were constructed and immunostained with D2-40 and antibodies to Prox-1 and VEGFR-3. Table 1 details the antibodies and conditions. A variety of normal tissue samples and neoplasms were included in the TMAs as controls. TMAs were utilized to facilitate screening of multiple tumours with multiple antibodies. In tumours showing cytoarchitectural heterogeneity, tissue cores were selectively taken to sample for variation. In addition, whole block tissue sections were stained in 23 tumours. These included nine tumours that were underrepresented in the TMA, such as ones in which the core sections were lost in processing. Cytoplasmic immunoreactivity was considered positive for D2-40 and VEGFR-3, whereas nuclear reactivity was considered positive for Prox-1. For 14 tumours with mixed well and poorly differentiated areas, selected whole blocks were stained to analyse for variation in patterns of reactivity based upon histological grade. A separate TMA block containing five lymphangiomas, four haemangiomas and six Kaposi sarcomas was used for antibody validation.

Correlative analyses between tumours having hobnail cytology and/or kaposiform architecture (morphological features often attributed to tumours with lymphatic differentiation) and immunopositivity with D2-40, Prox-1 and/or VEGFR-3 were performed using

Table 1. Antibodies and procedures

\begin{tabular}{llllll}
\hline Antibody & Species & Manufacturer & Address & Dilution & Pretreatment \\
\hline D2-40 & Mouse & Signet & Dedham, MA, USA & $1: 50$ & None \\
\hline Prox-1 & Rabbit & Abcam & Cambridge, MA, USA & $1: 100$ & HIER pH 6 \\
\hline VEGFR-3 & Rabbit & Abcam & Cambridge & $1: 150$ & HIER pH 6 \\
\hline
\end{tabular}

HIER, heat-induced epitope retrieval. 
histograms and Fisher's exact test. A $P$-value of $<0.05$ was considered statistically significant.

\section{Results}

Among the 49 angiosarcomas, 22 were sporadic cutaneous tumours (18 of the scalp or face, and one each on the back, ante cubit, vulva and buttock), 15 were sporadic visceral/deep-seated tumours (five breast, three heart, and one each involving mediastinum, thigh, adrenal gland, retroperitoneum, aorta, maxillary sinus, and femur), eight were radiationassociated (five breast, one scrotum, one suprapubic skin, and one oral mucosa) and four were lymphoedema-associated (all involving the lower extremity). Nineteen tumours had well-differentiated areas, 42 had moderately or poorly differentiated areas. Fourteen tumours had both well and poorly differentiated areas within the same tumour. Architectural features were vasoformative in 38 , solid in 28 , and kaposiform/fascicular in 11. Cytological features were epithelioid in 22 , hobnail in 19 , spindled in 16 , and pleomorphic in five. Many tumours showed morphological heterogeneity in terms of architectural and cytological features and were therefore included in more than one group in each category.

Twenty-six tumours (53\%) were positive for D2-40, 37 (76\%) for Prox-1 and 28 (57\%) for VEGFR-3. Of the 14 tumours with mixed well and poorly differentiated areas in which whole block sections were immunoreactive, there was no variation in reactivity based upon grade. Of the validation samples, five of five lymphangiomas were positive for D2-40 and Prox-1, while four were positive for VEGFR-3. All four haemangiomas were negative for D2-40 and VEGFR-3, while one was positive for Prox-1. Among the six Kaposi sarcomas, five were positive with D2-40, five for Prox-1 and five for VEGFR-3 (Table 2).

Tumours with hobnail cytology were more likely to express lymphatic antigens than those without hobnailing (Figure 1). Seventy-nine percent with hobnailing were positive for D2-40 compared with 37\% without hobnailing, and the difference was statistically significant $(P=0.01)$. Eighty-nine percent of tumours with hobnailing were positive for Prox-1 compared with $67 \%$ without, and $68 \%$ were positive for VEGFR-3 compared with $50 \%$ without. These trends were not statistically significant $(P=0.09$ and $P=0.25$ for Prox-1 and VEGFR-3, respectively). Comparing tumours with and without kaposiform areas, kaposiform tumours were more often positive for Prox-1 (91\% compared with 71\%) and VEGFR-3 (73\% compared with 53\%), but less often positive for D2-40 (45\%
Table 2. Immunohistochemical results of validation samples

\begin{tabular}{llll}
\hline Antibody & Haemangioma & Lymphangioma & $\begin{array}{l}\text { Kaposi's } \\
\text { sarcoma }\end{array}$ \\
\hline D2-40 & $0 / 4$ & $5 / 5$ & $5 / 6$ \\
\hline Prox-1 & $1 / 4$ & $5 / 5$ & $5 / 6$ \\
\hline VEGFR-3 & $0 / 4$ & $4 / 5$ & $5 / 6$ \\
\hline
\end{tabular}

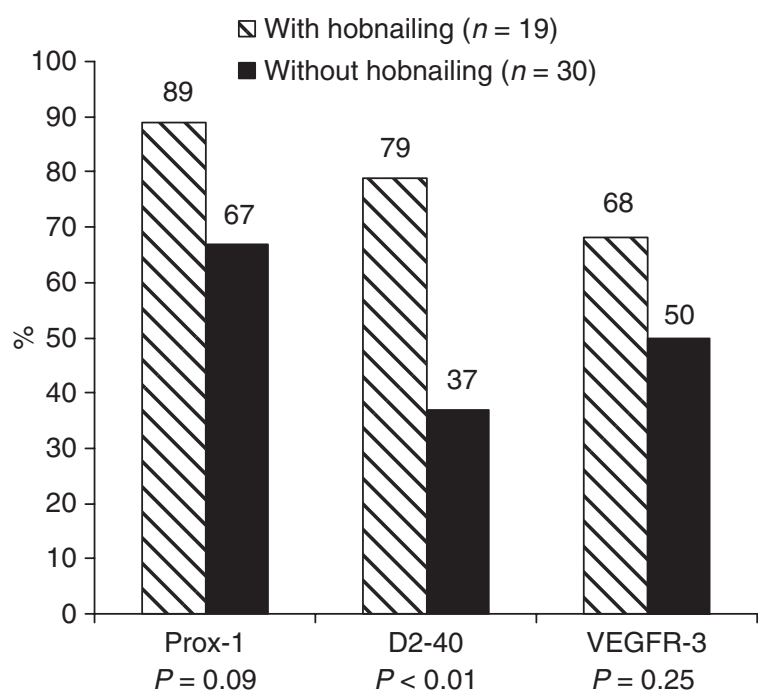

Figure 1. Histogram comparing tumours with and without hobnail endothelial cells. D2-40 is significantly more often positive, whereas Prox-1 and VEGFR-3 show a trend towards staining tumours with hobnailing.

compared with 55\%). None of these differences was statistically significant.

Among angiosarcomas with hobnail cytology, we identified a morphologically distinctive subgroup of 10 well-differentiated cutaneous tumours composed of irregular interanastomosing channels, mostly devoid of red blood cells, lined by endothelial cells with prominent hobnailing (Figures 2 and 3). These tumours also had prominent lymphocytic aggregates (Figure 4), some with germinal centres. Within this subgroup were six men and four women, median age 76 years (range 49-82 years). Nine were sporadic cutaneous tumours of the scalp and face and one was a lymphoedema-associated tumour of the lower extremity.

All 10 tumours in this subgroup were positive for D2-40 and Prox-1, and six were positive for VEGFR-3 (Figure 5). These tumours were significantly more likely to be positive for D2-40 $(P=0.01)$ and were more likely, but not statistically significantly so, to be 


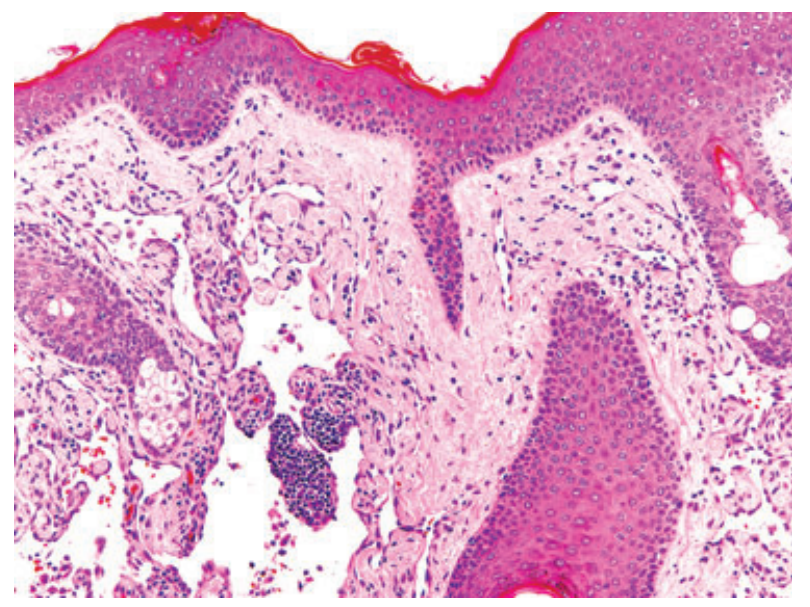

Figure 2. Ten tumours have histological features suggestive of lymphatic differentiation. All have well-differentiated areas composed of interanastomosing channels largely devoid of red blood cells and prominent lymphoid aggregates.
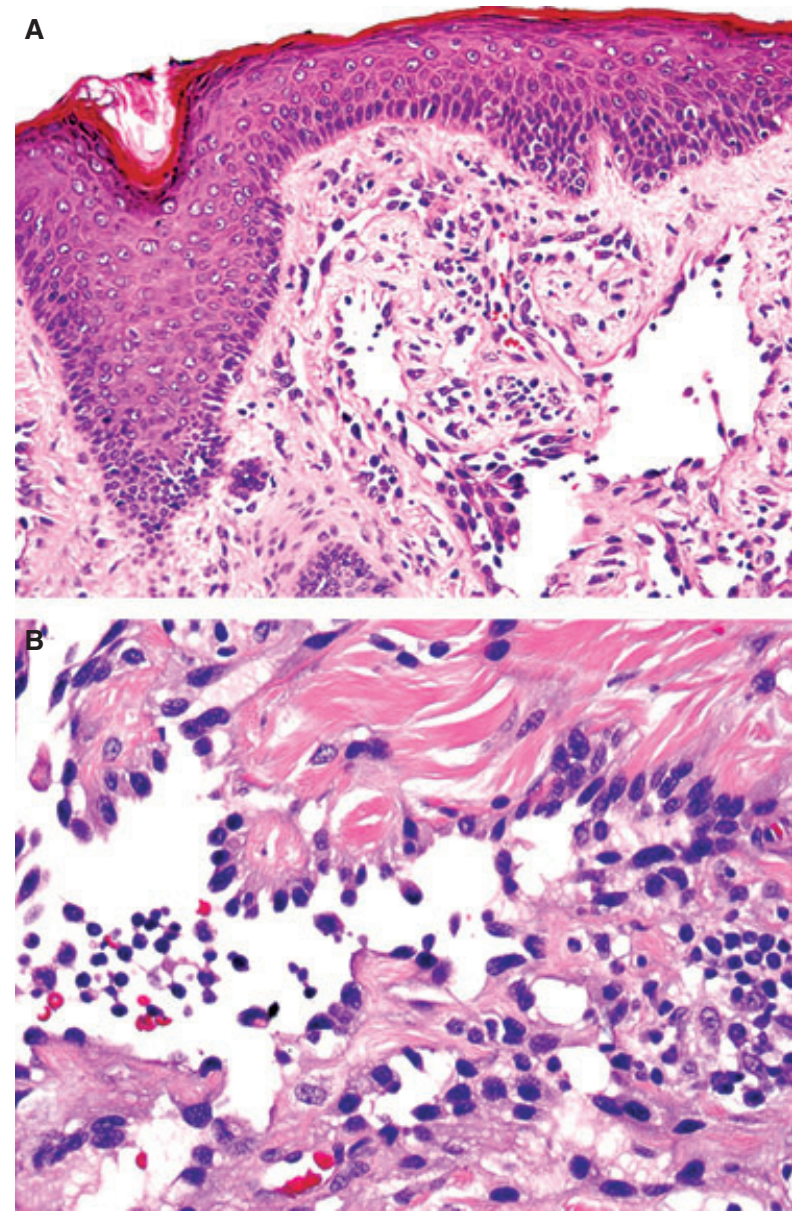

Figure 3. All 10 tumors have prominent hobnail endothelial cells characterized by cuboidal cells with scant cytoplasm and apical nuclei protruding into the lumen. (A, Low power; B, high power.)

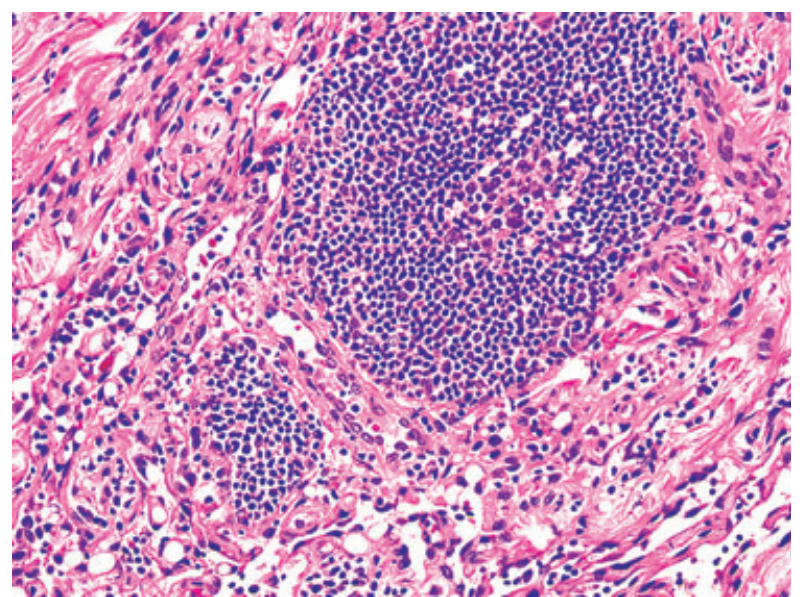

Figure 4. Lymphocytic infiltrates are prominent in all 10 'lymphangiosarcomas,' including frequent lymphoid aggregates.

positive for Prox-1 ( $P=0.09)$ than the other tumours in our series (Figure 6). By evaluating multi-antigen expression, six tumours in this subgroup $(60 \%)$ expressed all three antigens, compared with only 39\% of the other tumours in our series $(P=0.29)$. Furthermore, all 10 tumours in this subgroup were positive for D2-40 and Prox-1, whereas only 41\% of other tumours were positive for both, a statistically significant difference $(P=0.03)$. All 10 of these tumours had prominent lymphoid aggregates compared with only $51 \%$ of the remaining tumours, a significant difference $(P=004)$. Two of the 10 tumours were purely welldifferentiated angiosarcomas, whereas eight had additional poorly differentiated areas (Figure 7). In three tumours the poorly differentiated areas were epithelioid (Figure 8), in two they were kaposiform/fascicular, in one there were both epithelioid and kaposiform/ fascicular areas, and in one the high-grade area was non-epithelioid. As in all tumours with mixed-grade histology in our series, the poorly differentiated areas showed the same antigen profile as the well-differentiated areas in these 10 tumours.

At last follow-up (mean 7 years, range 8 months to 9 years), $60 \%$ of these 10 patients had died of disease, $20 \%$ were alive with disease, one had died of a cause not known to us, and one was alive with no evidence of disease after 9 years. By comparison, among the 39 cases not included in this subgroup $57 \%$ had died of disease, $5 \%$ were alive with disease, $19 \%$ were alive with no evidence of disease, $8 \%$ were alive but their disease status was not known to us, and $11 \%$ were lost to followup. Clinicopathological and immunohistochemical features comparing the 10 cases with lymphangiosarcomatous differentiation with the remaining cases are summarized in Table 3. 


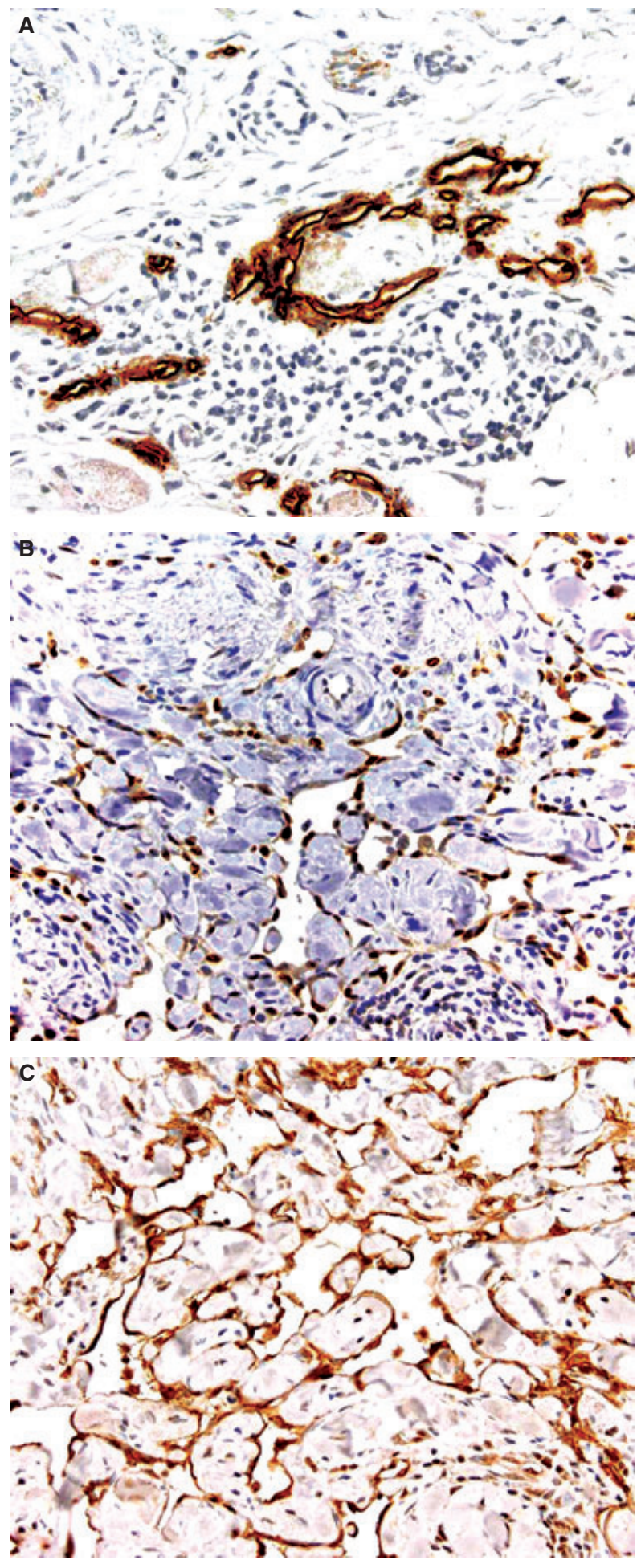

Figure 5. D2-40 (A) and Prox-1 (B) are positive in all 10 'lymphangiosarcomas', whereas six are positive for VEGFR-3 (C).

Of the four tumours in this study that arose within a setting of chronic lymphoedema, only one had prominent hobnailing and was included in the

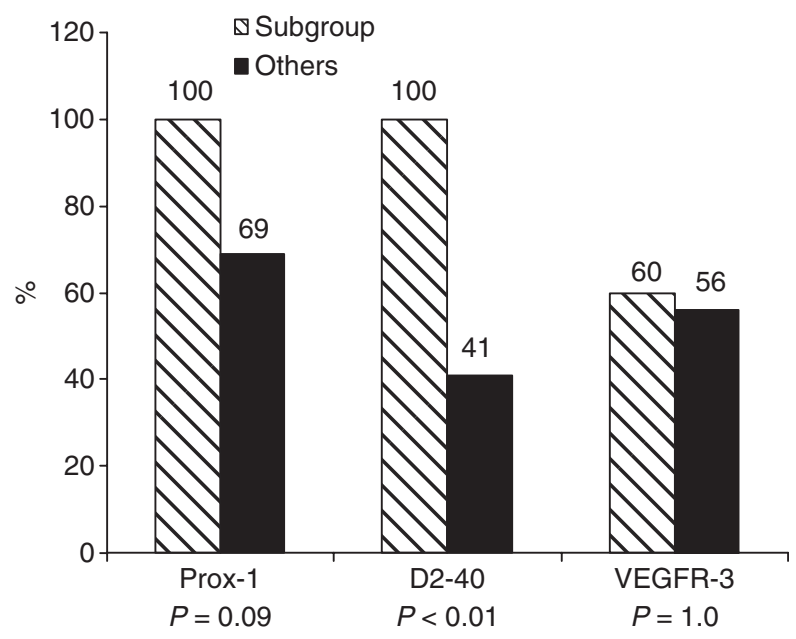

Figure 6. Histogram comparing the subgroup of tumours with prominent lymphatic differentiation by histology with the other tumours. D2-40 was significantly more often positive in the subgroup, whereas Prox-1 showed a trend toward more frequent immunoreactivity, and VEGFR-3 showed no trend.

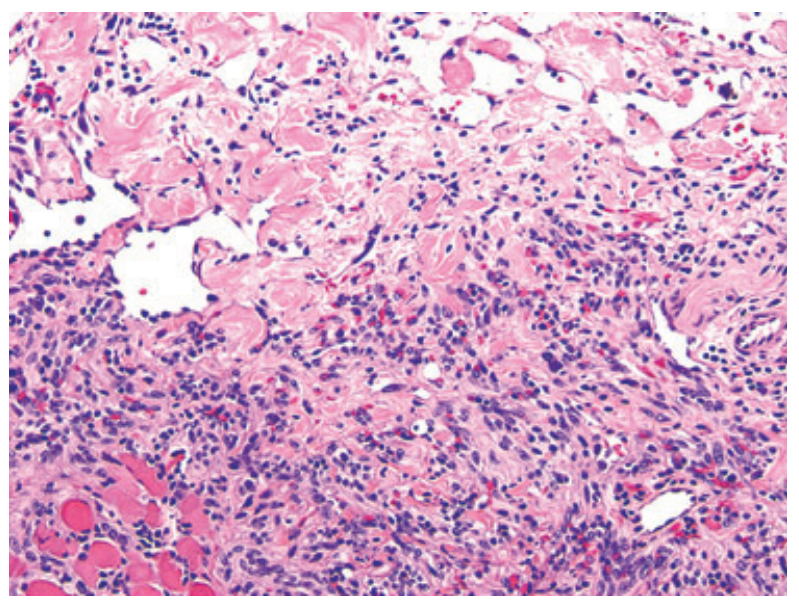

Figure 7. Eight of the 10 'lymphangiosarcomas' have poorly differentiated areas in addition to well-differentiated areas exemplified by this tumour. Note the low-grade vasoformative area juxtaposed to the high-grade solid area beneath it.

subgroup of 10 cases just discussed. It was positive for all three markers. The other three oedema-associated tumours were high-grade vasoformative and solid tumours. One was negative for all three markers, one expressed D2-40 only, and one expressed Prox-1 and VEGFR-3.

\section{Discussion}

Substantial progress has been made in our understanding of how the lymphatic vasculature is formed and remodelled with recent discoveries of molecular 


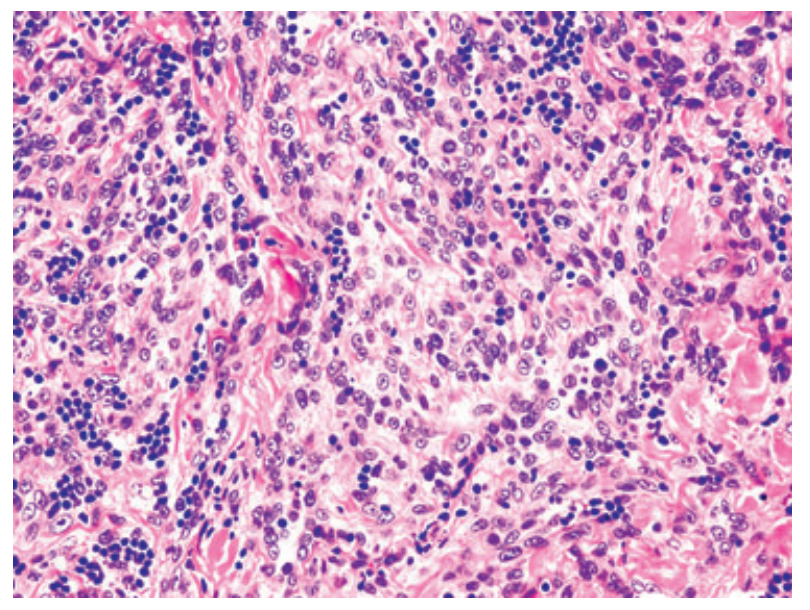

Figure 8. Most often the high-grade areas are epithelioid angiosarcomas.

mechanisms involved in lymphangiogenesis. The current model of lymphatic development theorizes that lymphatic endothelial cells originate from specific locations in the embryonic (or cardinal) vein, which migrate to form lymphatic sacs, and then undergo further sprouting and remodelling to form the lymphatic system. ${ }^{16}$ All endothelial cells in the embryonic vein express VEGFR-3. The earliest known event in lymphangiogenesis is polarized expression of the homeobox transcription factor Prox-1, which commits the cell to lymphatic differentiation. ${ }^{17}$ As lymphatics develop, their endothelial cells continue to express VEGFR-3 and Prox-1, then add LYVE-1 (a homologue of CD44), and finally podoplanin, a small transmembrane mucinlike protein ${ }^{16}$ detected with the antibody D2-40. By contrast, as blood vessels mature, they lose VEGFR-3 expression. Thus, in normal adult tissues lymphatics express VEGFR-3, Prox-1, LYVE-1 and podoplanin, whereas blood vessels usually express none of these antigens.

The specificity of lymphatic endothelial markers for neoplasms with lymphatic differentiation has been evaluated in a number of immunohistochemical studies. For example, D2-40 has been shown to be highly specific for distinguishing lymphangioma from haemangioma. ${ }^{3,5,6,9,12}$ VEGFR-3 has also been found to be highly expressed in lymphangiomas, but is less specific than D2-40 since it also stained substantial numbers of haemangiomas, 22 of 45 and 2 of 13, in two separate studies. ${ }^{4,11}$ Comparative studies of lymphangioma and haemangioma using LYVE-1 and Prox-1 are limited to only one study of each. LYVE-1 stained nine of nine lymphangiomas and 0 of 20 haemangiomas, ${ }^{8}$ while Prox-1 stained five of six lymphangiomas and 5 of 12
Table 3. Clinicopathological and immunohistochemical comparisons between cases with and without lymphangiosarcomatous differentiation

\begin{tabular}{|c|c|c|}
\hline & $\begin{array}{l}\text { Cases with } \\
\text { lymphangio- } \\
\text { sarcomatous } \\
\text { differentiation } \\
(n=10)\end{array}$ & $\begin{array}{l}\text { Others } \\
(n=39)\end{array}$ \\
\hline \multicolumn{3}{|l|}{ Clinical features } \\
\hline Sporadic cutaneous & $90 \%$ & $33 \%$ \\
\hline Scalp/face & $(90 \%)$ & $(23 \%)$ \\
\hline Sporadic visceral/deep & & $38 \%$ \\
\hline Breast & & $(13 \%)$ \\
\hline Visceral organ & & $(10 \%)$ \\
\hline Deep soft tissue & & $(10 \%)$ \\
\hline Bone & & $(3 \%)$ \\
\hline Lymphoedema-associated & $10 \%$ & $8 \%$ \\
\hline Radiation-associated & & $20 \%$ \\
\hline \multicolumn{3}{|l|}{ Microscopic features } \\
\hline Hobnail cytology & $100 \%$ & $23 \%$ \\
\hline Lymphoid aggregates & $100 \%$ & $51 \%$ \\
\hline $\begin{array}{l}\text { Kaposiform } \\
\text { architecture }\end{array}$ & $20 \%$ & $28 \%$ \\
\hline Epithelioid cytology & $40 \%$ & $46 \%$ \\
\hline \multicolumn{3}{|l|}{ Immunohistochemistry } \\
\hline D2-40+ & $100 \%$ & $41 \%$ \\
\hline Prox-1+ & $100 \%$ & $69 \%$ \\
\hline VEGFR-3+ & $60 \%$ & $56 \%$ \\
\hline \multicolumn{3}{|l|}{ Outcome } \\
\hline NED & $10 \%$ & $19 \%$ \\
\hline AWD & $20 \%$ & $5 \%$ \\
\hline DOD & $60 \%$ & $57 \%$ \\
\hline
\end{tabular}

NED, no evidence of disease; AWD, alive with disease; DOD, died of disease.

haemangiomas. ${ }^{7}$ In our current analysis of D2-40, VEGFR-3 and Prox-1, we observed high specificity for lymphangioma over haemangioma with all three markers (Table 2).

Although the histogenesis of Kaposi's sarcoma is not entirely clear, most investigators regard it as having at 
least partial lymphatic differentiation. Immunohistochemical studies of D2-40, VEGFR-3, Prox-1 and LYVE-1 have demonstrated near $100 \%$ sensitivity rates in Kaposi's sarcoma. ${ }^{3-5,8,11,13}$ Similarly, we found high rates of immunoreactivity in Kaposi's sarcoma, with five of six tumours staining with at least one of the three markers we evaluated (Table 2). Thus, based upon our results and those from other studies, D2-40, Prox-1 and VEGFR-3 appear to be rather specific markers of lymphatic neoplasms.

A number of immunohistochemical studies of lymphatic differentiation in angiosarcoma have been published, ${ }^{3-5,8,11,14,15,18,19}$ most of which have employed only a single lymphatic endothelial-specific antibody. Although most of these studies conclude that angiosarcomas frequently show mixed lymphatic and blood vascular endothelial differentiation, some suggest that there may be subsets with at least partial lymphatic differentiation, ${ }^{11,15}$ including some that might be considered lymphangiosarcomas. ${ }^{5}$

In the largest series of such studies, Partanen et al. ${ }^{4}$ examined 60 angiosarcomas for VEGFR-3, expression and found $48(80 \%)$ to be positive. They observed that epithelioid tumours were less often positive, but concluded that VEGFR-3 lacks specificity for lymphatic differentiation. By contrast, Folpe et al. ${ }^{11}$ also staining for VEGFR-3 found 8 of 16 angiosarcomas to be positive, and correlated immunopositivity with hobnail histology and lymphocytic infiltration. They concluded that angiosarcomas expressing this receptor protein may represent tumours displaying lymphatic differentiation. Similarly, in a recent study Itakura et al. ${ }^{15}$ examined 34 angiosarcomas for multiple VEGFs and VEGFRs. They found 27 (79\%) to stain for VEGFR-3 and, based upon all their results, concluded that angiosarcoma of the scalp may include examples of lymphatic origin.

There are three studies of anti-podoplanin or D2-40 in angiosarcoma. In the earliest study, BreitenederGeleff et al. ${ }^{14}$ found 10 of 11 angiosarcomas to be positive for podoplanin, and concluded that most angiosarcomas show a mixed phenotype. By contrast, Kahn et al. ${ }^{5}$ found three of seven angiosarcomas to stain with D2-40. They observed that immunopositivity correlated with hobnail histology and lymphoid aggregates, and concluded that a subset of angiosarcomas can undergo at least partial differentiation along the lymphatic endothelial lineage and could be classified as lymphangiosarcomas. More recently, Fukunaga et al. ${ }^{3}$ found 7 of 15 angiosarcomas to be D2-40+, which correlated with epithelioid and papillary endothelial cells, and concluded that there may be a subset of angiosarcoma with dual vascular and lymphatic endothelial phenotypes.
Only one study of LYVE-1 expression in angiosarcoma has been published, ${ }^{8}$ in which all 10 tumours were positive. The authors concluded that angiosarcomas show characteristics of both lymphatic and blood vessel phenotypes. An older study from 1987 by Holden et al. ${ }^{19}$ using periodate-lysine paraformaldehyde fixative and the antibodies to EN4, PAL-E and factor VIIIrelated antigen and electron microscopy concluded that based upon their marker results, angiosarcoma of the face and scalp can be interpreted as consistent with being of lymphatic derivation, but in contradistinction, their ultrastructural studies showed features suggestive of blood vascular differentiation. Finally, although Prox-1 has been examined in benign vascular neoplasms, we are not aware of any study beside our own in angiosarcoma.

In our series we found VEGFR-3, D2-40 and Prox-1 immunoreactivity in 57,53 and $76 \%$ of our tumours, respectively, confirming that lymphatic differentiation is common in angiosarcoma. We also observed that immunopositivity correlated with certain morphological features that are often attributed to lymphatic differentiation. Specifically, there was a trend for tumours with hobnail cytology and kaposiform architecture to be positive with these markers, including a statistically significant association between D2-40 reactivity and hobnailing.

Armed with these results, we identified 10 angiosarcomas that had evidence of lymphatic differentiation, both histological and immunohistochemical, which may be deserving of the appellation lymphangiosarcoma. All were cutaneous tumours, nine occurring on the scalp or face of elderly adults and one in association with chronic lymphoedema on the lower extremity. Microscopically, all 10 tumors had well-differentiated areas comprising interanastomosing channels that were largely devoid of red blood cells and lined by endothelial cells with prominent hobnailing, had lymphoid aggregates, and expressed multiple lymphatic antigens. Furthermore, these tumours were significantly more likely to express D2-40 and Prox-1. However, our case numbers are probably too low to draw definitive conclusions from this statistic alone.

Interestingly, we are not the first to suggest that lymphangiosarcoma may arise outside of the setting of lymphoedema or that it has a predilection for the scalp and face. For example, Reed et al. in $1966^{20}$ reported six cases of lymphangiosarcoma of the scalp. These cases were morphologically similar to our cases, with welldifferentiated areas showing hobnailing and lymphoid aggregates. Some of their cases also had poorly differentiated epithelioid and spindle cell areas, as we observed in 8 of our 10 tumours. Sordillo et al. in $1981^{21}$ reported 
the clinicopathological findings in 44 lymphangiosarcomas. This series consisted of 40 tumours associated with chronic lymphoedema and four scalp tumours not associated with lymphoedema. They described cuboidal and spindled endothelial cells lining capillary spaces without red blood cells, as well as areas resembling Kaposi's sarcoma and higher grade solid areas. More recently, Hancox et al. in 2006 reported a case of lymphangiosarcoma of the cheek that had hobnailing, a lymphoplasmacytic infiltrate and D2-40 staining. They concluded this tumour should be classified as an angiosarcoma of mixed histology with lymphatic differentiation predominating.

The 10 cases of 'lymphangiosarcoma' in our series had similar poor outcomes compared with the rest of the group. Thus, it not clear whether separating them from other angiosarcomas is clinically important, but with only 10 cases we would not have detected subtle differences in behaviour. In addition, although there are currently no specific therapies directed to tumours with lymphatic differentiation, it is conceivable that specific targeted therapies might be developed in the future.

In summary, based upon immunohistochemical results, angiosarcomas often show evidence of lymphatic differentiation. This may be explained by recapitulation of the phenotypic plasticity evidenced by embryonic endothelial development. Furthermore, there appears to be a subset of angiosarcomas, which, based upon morphology and immunohistochemistry, show more prominent lymphatic differentiation than others. Such tumours have a predilection for the skin of the scalp and face, and are usually not associated with chronic lymphoedema. Thus, the concept of lymphangiosarcoma, which was originally based upon clinical findings, may be evolving towards a pathological definition.

\section{References}

1. Stewart FW, Treves N. Lymphangiosarcoma in postmastectomy edema: a report of six cases in elephantiasis chirugica. Cancer 1948; 1; 64-81.

2. Weiss SW, Goldblum JR. Enzinger and Weiss's soft tissue tumors, 5th edn. Philadelphia: Mosby, 2008.

3. Fukunaga M. Expression of D2-40 in lymphatic endothelium of normal tissues and in vascular tumours. Histopathology 2005; 46; 396-402.

4. Partanen TA, Alitalo K, Miettinen M. Lack of lymphatic vascular specificity of vascular endothelial growth factor receptor 3 in 185 vascular tumors. Cancer 1999; 86; 2406-2412.

5. Kahn HJ, Bailey D, Marks A. Monoclonal antibody D2-40, a new marker of lymphatic endothelium, reacts with Kaposi's sarcoma and a subset of angiosarcomas. Mod. Pathol. 2002; 15; 434-440.
6. Galambos C, Nodit L. Identification of lymphatic endothelium in pediatric vascular tumors and malformations. Pediatr. Dev. Pathol. 2005; 8; 181-189.

7. Reis RM, Reis-Filho JS, Longatto Filho A, Tomarev S, Silva P, Lopes JM. Differential Prox-1 and CD 31 expression in mucousae, cutaneous and soft tissue vascular lesions and tumors. Pathol. Res. Pract. 2005; 201; 771-776.

8. Xu H, Edwards JR, Espinosa O, Banerji S, Jackson DG, Athanasou NA. Expression of a lymphatic endothelial cell marker in benign and malignant vascular tumors. Hum. Pathol. 2004; 35; $857-$ 861.

9. Arai E, Kuramochi A, Tsuchida T et al. Usefulness of D2-40 immunohistochemistry for differentiation between kaposiform hemangioendothelioma and tufted angioma. J. Cutan. Pathol. 2006; 33; 492-497.

10. Lymboussaki A, Partanen TA, Olofsson B et al. Expression of the vascular endothelial growth factor $\mathrm{C}$ receptor VEGFR-3 in lymphatic endothelium of the skin and in vascular tumors. Am. J. Pathol. 1998; 153; 395-403.

11. Folpe AL, Veikkola T, Valtola R, Weiss SW. Vascular endothelial growth factor receptor-3 (VEGFR-3): a marker of vascular tumors with presumed lymphatic differentiation, including Kaposi's sarcoma, kaposiform and Dabska-type hemangioendotheliomas, and a subset of angiosarcomas. Mod. Pathol. 2000; 13; $180-185$.

12. Debelenko LV, Perez-Atayde AR, Mulliken JB, Liang MG, Archibald TH, Kozakewich HP. D2-40 immunohistochemical analysis of pediatric vascular tumors reveals positivity in kaposiform hemangioendothelioma. Mod. Pathol. 2005; 18; 1454-1460.

13. Jussila L, Valtola R, Partanen TA et al. Lymphatic endothelium and Kaposi's sarcoma spindle cells detected by antibodies against the vascular endothelial growth factor receptor-3. Cancer Res. 1998; 58; 1599-1604.

14. Breiteneder-Geleff S, Soleiman A, Kowalski H et al. Angiosarcomas express mixed endothelial phenotypes of blood and lymphatic capillaries: podoplanin as a specific marker for lymphatic endothelium. Am. J. Pathol. 1999; 154; 385-394.

15. Itakura E, Yamamoto H, Oda Y, Tsuneyoshi M. Detection and characterization of vascular endothelial growth factors and their receptors in a series of angiosarcomas. J. Surg. Oncol. 2008; 97; 74-81.

16. Makinen T, Norrmen C, Petrova TV. Molecular mechanisms of lymphatic vascular development. Cell. Mol. Life Sci. 2007; 64; 1915-1929.

17. Tammela T, Petrova TV, Alitalo K. Molecular lymphangiogenesis: new players. Trends Cell Biol. 2005; 15; 434-441.

18. Hancox JG, Yount LS, Eaton JS, Barnette DJ, Greenway HT Jr. Lymphangiosarcoma presenting as asymptomatic swelling of the cheek. J. Am. Acad. Dermatol. 2007; 56; 530-531.

19. Holden CA, Spaull J, Das AK, McKee PH, Jones EW. The histogenesis of angiosarcoma of the face and scalp: an immunohistochemical and ultrastructural study. Histopathology 1987; $11 ; 37-51$.

20. Reed RJ, Palomeque FE, Hairston MA 3rd, Krementz ET. Lymphangiosarcomas of the scalp. Arch. Dermatol. 1966; 94; 396-402.

21. Sordillo PP, Chapman R, Hajdu SI, Magill GB, Golbey RB. Lymphangiosarcoma. Cancer 1981; 48; 1674-1679. 